\title{
The Potential of Cold Plasma for Safe and Sustainable Food Production
}

\author{
Paula Bourke \\ Technological University Dublin, paula.bourke@tudublin.ie \\ Dana Ziuzina \\ Technological University Dublin, dana.ziuzina@tudublin.ie \\ Daniela Boehm \\ Technological University Dublin, daniela.boehm@tudublin.ie
}

See next page for additional authors

Follow this and additional works at: https://arrow.tudublin.ie/scschbioart

Part of the Biology Commons

\section{Recommended Citation}

Bourke, P., Ziulina, D., Boehm, D., Cullen, P.J. \& Keener, K. (2018). The potential of cold plasma for safe and sustainable food production Trends in Biotechnology, 36(6), pp.615-626. doi:10.1016/

j.tibtech.2017.11.001

This Article is brought to you for free and open access by the School of Biological Sciences at ARROW@TU Dublin. It has been accepted for inclusion in Articles by an authorized administrator of ARROW@TU Dublin. For more information, please contact arrow.admin@tudublin.ie, aisling.coyne@tudublin.ie,gerard.connolly@tudublin.ie.

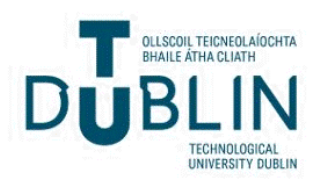




\section{Authors}

Paula Bourke, Dana Ziuzina, Daniela Boehm, Patrick Cullen, and Kevin Keener 
1 Title: The potential of cold plasma for safe and sustainable food production

2 Authors: Paula Bourke ${ }^{1}$, Dana Ziuzina ${ }^{1}$, Daniela Boehm ${ }^{1}$, Patrick J. Cullen ${ }^{2}$, Kevin Keener ${ }^{3}$

3

4 1. Plasma Research Group, School of Food Science and Environmental Health, Dublin

5 Institute of Technology, Dublin 1, Ireland

6 2. Department of Chemical and Environmental Engineering, University of Nottingham,

7 Nottingham, NG7 2RD

8 3. Centre for Crops Utilization Research, Bio-Century Research Farm, Iowa State University,

9 1041 Food Sciences Building, Ames, IA 50011-1061

10

Corresponding Author: paula.bourke@dit.ie

13 ESHI Twitter: @ESHI_DIT

\section{Abstract}

Cold plasma science and technology is increasingly investigated for translation to a plethora of issues presenting in the agriculture and food sectors. The diversity of the mechanisms of action of cold plasma and the flexibility as a standalone technology or one that can integrate with other technologies, provide a rich resource for driving innovative solutions. The emerging understanding of the longer term role of cold plasma reactive species and follow on effects across a range of systems will provide understanding of how cold plasma may be optimally applied to biological systems in agricultural and food sectors. Here we present the current status, emerging issues, regulatory context and opportunities with respect to the broad stages of primary and secondary food production. 

production.

There are many reasons that lead to increased demand for innovative sustainable technologies for the agriculture and food sectors. These include an increasing global population leading to increased demand for food, water and energy resources. Inputs to agriculture and food production are under regulatory review in many regions in terms of sustainability, human safety and long term eco-safety. Many of the persistent contamination, spoilage and safety issues in the agriculture and food sectors are microbially derived, thus a parallel pressure emanates from the issue of antimicrobial resistance, and alternative approaches for microbial control across the agriculture and food sectors, as well as healthcare, are required. The safety of foods in terms of their immune reactivity is increasingly important, and food producers seek ways to mitigate or reduce allergenicity either in foods or in processing environments. Atmospheric cold plasma is a flexible approach with demonstrated efficacy for control of many risks across these sectors, and has the potential to provide transformative and sustainable technology interventions. This potential is based on the combination of increased knowledge of mechanisms and interactions with known risks and advances in engineering and design.

Thus, this review presents the current status, issues and opportunities in relation to applying cold plasma to the agriculture and food continuum (Figure 1, Key Figure). The regulatory status from a European and United States perspective is discussed to provide insights for the adoption of this technology. Cold plasma (CP) (see Glossary) technology has been explored for a number of applications at multiple stages of primary and secondary food production, including treatment of raw materials, intermediate or finished products and treatment of the processing equipment, facilities and environment, due to the abundance of advantages it presents. These advantages include operation at low temperatures, short processing times, energy efficiency and high antimicrobial efficacy with minimal impact on food quality and the environment (Figure 2).

\section{Primary production}

\section{Antimicrobial control}

Primary production encompasses the growing, cultivation, harvesting or collection of food. 
consumption or to turn them into other products. This includes transporting, sorting, cleaning, blending, and milling. Foods may then also undergo secondary processing, where they are transformed into new products." In the primary stages of food production, atmospheric pressure $\mathrm{CP}$ has been successfully applied to inactivate a wide range of microorganisms, including foodborne pathogenic bacteria and fungi, and spoilage microorganisms on grains and seeds or crops intended for sowing or storage.

In the past 5 years, a diverse range of CP laboratory scale systems with various application modes (Box 1) have been tested for surface decontamination of seeds of dill, carrot, parsley, wheat, pepper corns, alfalfa, onion, radish, cress seed, chickpea, rapeseed and maize and wheat grains $[2-7]$.

The characteristics of target microorganisms play an important role in achieving successful decontamination with $\mathrm{CP}$ technology. Higher inactivation rates are achieved for monospecies surface inoculations than for seed native microflora, which presents as multispecies microbial communities that are distributed on the surface as well as within internal seed structures [5]. Dasan and colleagues [8] reported higher resistance of fungal spores inoculated on maize grains as compared to bacterial cells, which was related to the differences in cytology, morphology, reproductive cycles and growth.

Besides high antimicrobial potential, any decontamination technology including CP should retain and/or improve the physicochemical and physiological properties of seeds to ensure that high quality seeds are provided for growers and consumers. However, in recent investigations, this combination of achieving maximal microbial reductions and significant improvements in seed quality parameters has not always been studied or demonstrated in tandem. Unfortunately, increases in treatment duration and/or input power required to obtain the highest antimicrobial efficiency (Box 2) results in inhibiting germination and the growth potential of seeds [5-7,9]. The potential of $\mathrm{CP}$ technology to improve physicochemical (hydrophobicity, wettability, moisture content, enzymatic activity, protein concentration, chlorophyll, nitrogen and soluble phenol content) and physiological (germination, growth, vigour, fresh weight and overall yield) parameters of various seeds during different plant formation stages has been demonstrated in a number of studies that do not focus on the microbiological safety aspects of seeds. Importantly, for achieving enhanced seed growth parameters, the plasma chemical composition and treatment dose (in terms of the working 
gas, power input and treatment time) should be adequately selected, controlled and evaluated with respect to each individual type of seed intended for treatment.

The duration of treatment is one of the most important plasma treatment parameters investigated to date and, depending on the system design and voltage levels used for generation of plasma, it may vary widely (5 s - $30 \mathrm{~min}$ ). In most of the cases, shorter treatment durations can significantly improve seed growth parameters, while extended treatments may have inhibitive effects on seeds [9-13].

The response of seeds to plasma treatment was shown to depend on plasma gas composition: apparent (wheat) seed growth inhibition symptoms occurred in samples exposed to nitrogen plasma than in samples exposed to helium plasma, attributed to the higher NO content [14].

Stimulatory effects of plasma treatment on germination and seedling growth characteristics of soybean and wheat vary with different CP treatment power levels $[15,16]$. Another important parameter that needs to be considered is the type of seed, since different seeds may respond differently to plasma treatment [17-19]. Moreover, differences in response to plasma treatment may occur among different cultivars within one plant taxon [11]. A positive effect of plasma treated water (PTW) and combination of treatment with plasma processed air and PTW on plant germination rates and seedling growth have been reported, however, the duration of plasma treatment has to be optimized for each type of seed individually $[19,21,22]$.

The interactions between plasma and growing plants may be complex, so the question remains whether long-term positive treatment effects could be also achieved, and if these relate predominantly to the manipulation of the plant micro-flora, plant biochemical responses or the plant growth medium.

The retention of nutritional characteristics of seeds is also of major importance, as it is known that plasma generated reactive species, especially ozone, can induce major changes in the physicochemical constituents of grains. For example, protein modification and/or lipid and starch oxidation can occur through multiple chemical reactions during the treatment [20]. To date, the influence of plasma treatment on seed or grain sensory attributes has rarely been reported. Puligundla and colleagues [7] reported that sprouts of plasma treated rapeseeds for up to $2 \mathrm{~min}$ did not exhibit undesirable sensory properties (appearance, color, flavor, taste, texture and overall acceptance) compared to sprouts of seeds treated for longer duration (3 min), where the quality was negatively affected. 
136 The potential applications of CP have been demonstrated for insect control in stored products.

137 El-aziz et al. [23] and Mohammadi et al. [24] demonstrated that CP treatment resulted in

138 significant increases in larval and pupal mortality and a decrease in adult emergence due to

139 the stress caused by the action of reactive oxygen species generated during the treatment.

$140 \quad$ Mycotoxin degradation

141 Due to its high oxidizing potential, CP technology has been successfully utilized for

142 degradation of mycotoxins that can contaminate seeds, grains or crops, thereby posing high

143 risks to human and animal health. However, the food matrix and the type of mycotoxin can

144 influence the efficacy of plasma [25]. Shi and colleagues [26] demonstrated that applying

145 higher levels of relative humidity with a modified gas with high oxygen content and post-

146 treatment storage could improve the efficacy of CP for the reduction of mycotoxins on seeds.

147 Applications of $\mathrm{CP}$ for pesticide degradation have been demonstrated for a number of

148 different organochlorine and organophosphorus pesticide compounds and on various

149 substrates. CP can furthermore be utilised for air pollution control and soil remediation (Box $1503)$.

151

\section{Secondary production}

153 The application of plasma treatment at secondary stages of food production can serve a range

154 of different purposes, such as improvement of food safety, extension of shelf-life,

155 maintaining quality and nutrition or improving processing. Of importance is the compatibility

156 of such technology with current practices, including the interaction with gases and packaging

157 being used. Cold plasma interactions with food packaging materials were recently

158 comprehensively presented by Pankaj and colleagues [27].

159 Decontamination for shelf-life extension

160 The globalization of the food market with increasing distances between the point of

161 production/processing and consumption along with mounting pressure to reduce food waste

162 and improve sustainability are driving forces behind efforts toward shelf-life extension in

163 particular for fresh produce and meat products. Due to its ability to inactivate

164 microorganisms, CP can delay food spoilage resulting from bacterial and fungal growth.

165 Investigations on improving food shelf-life through atmospheric cold plasma have considered

166 ready-to-eat foods such as fresh fruit and vegetables [28,29] and meat [30,31].

167 Microbiological interactions with cold plasma and mechanisms were recently reviewed by

168 Bourke and colleagues [32]. 
The food matrix and surface structure have strong influence on the efficacy of plasma based microbial decontamination. Cells in a liquid carrier were found to be more resistant to plasma inactivation than those on a solid like surfaces due to the need for reactive species to diffuse into the liquid [33]. Internalization of bacteria and bacterial biofilms in cracks, crevasses or structures such as plant leaf stomata can protect microorganisms against plasma-based inactivation [34]. The food structure hence needs to be taken into account in designing plasma treatments for food products to ensure efficacy and safety. Food intrinsic factors such as osmolarity and $\mathrm{pH}$ also affect the efficacy of plasma treatment as they can result in stress hardening of bacteria, making them more resistant [35], while lipid and protein content and antioxidant state can diminish the activity of plasma reactive species.

\section{Food quality}

Few studies have addressed the organoleptic acceptability of plasma-treated food products, and those that have are primarily focused on the impact of plasma on visual appearance and colour in particular. One of the few studies that included product consumption found no difference in sensory acceptability, namely appearance, colour, flavour, taste and texture, of dried squid shreds treated with corona discharge for microbial decontamination despite decreases in water content and increased lipid peroxidation [36]. A sensory evaluation of colour, freshness, firmness and texture of treated radicchio found no difference directly after treatment but scored the treated product at 2/10 in terms of overall acceptability after 1 and 3 days of storage [37]. Plasma treatment of pork meat was found to result in lighter meat colour but also greening possibly due to the reaction of myoglobin with plasma generated $\mathrm{H}_{2} \mathrm{O}_{2}$ [38] . The generation of ROS such as ozone could lead to a bleaching of produce colour and negatively impact the visual appearance. Fresh produce including tomatoes, carrots, and lettuce showed small but insignificant colour changes when exposed to plasma treatment [39] or plasma-activated water [40]. In fact, the inactivation of polyphenoloxidase (PPO) and peroxidase (POD) could prevent undesirable browning of fruit and vegetables [41]. In blueberries, treatment with plasma resulted in reduced concentrations of anthocyanin but produced a darker blue surface colour of the fruits [42].

Importantly, in a study of the nutritional parameters of orange juice, no significant effects of plasma treatment on its vitamin $\mathrm{C}$ content or its $\mathrm{pH}$, turbidity or Brix, were observed [43]. Antioxidant content (ascorbic acid polyphenols) and activity of minimally processed kiwifruit remained unaffected by plasma treatment [44] and the enzyme polyphenol oxidase was observed to be inhibited by plasma treatment. 
203 The use of plasma treatment for modification of the functionality of foods has been investigated. $\mathrm{CP}$ was found to modify the functionality of wheat flour by producing a stronger dough and accelerating lipid peroxidation [45]. Plasma treatment of brown rice resulted in reduced cooking time by increasing grain hydrophilicity [46] and rice starch showed modified rheological properties [47]. The inactivation of enzymes involved in the browning of fruit and vegetables through plasma exposure can maintain food quality and consumer acceptability over storage time.

\section{Food Safety}

\section{Biological/chemical changes}

A range of chemical changes to food components have been reported, including the oxidation of sugars to organic acids, the modification of amino acid residues in proteins [48], loss of protein structure [49], and the peroxidation of lipids and unsaturated fatty acids in particular [50]. The inactivation of enzymes such as polyphenoloxidase (PPO) and peroxidase (POD) in response to plasma treatment result from the disruption of the alpha-helical structure [41].

\section{Potential toxicity}

In depth investigations of the safety of plasma for food applications remain lacking. Plasmatreated solutions, ranging from simple PTW to complex compositions containing carbohydrates, lipids or proteins, have demonstrated cytotoxic activity in mammalian cell models, which has sparked interest in their use for cancer treatment [51]. Mutagenic effects of protein-treated solutions have been observed in some studies but not in others [52-55] and highlight the complexity resulting from the range of diverse plasma devices, treatment regimens and target substances.

These investigations have been performed in the context of medical applications of plasma, and while they highlighted the need for a better understanding of the effect of plasma-treated substances, studies focused more directly on food-related toxicity are needed. Of significance here are evaluations of the persistence of cytotoxic effectors in food over time, their concentration and their oral toxicity, which should be evaluated similarly to other decontaminants used in food processing including chlorine or ozone. The potential of adverse effects resulting from plasma-induced changes to food constituents must therefore be addressed and contextualized.

In view of the generation of substantial concentrations of nitrite and/or nitrate in plasmatreated solutions, which can reach the $\mathrm{mM}$ range depending on plasma device and treatment parameters [56], the potential accumulation of nitrogen compounds in food products needs to be considered. In fact, plasma-treated water has been tested as an alternative source of nitrite 
for the curing of sausages [57]. Nitrite and nitrate concentrations should be monitored in foods, subjected to either direct plasma-treatment or washing with plasma-activated water, to ensure acceptable levels and also to avoid a critical build-up in processing effluent, which could be of environmental concern. The concentrations generated in plasma treated water can in fact exceed WHO outlined safety guidelines for drinking water of $50 \mathrm{mg} / \mathrm{l}$ nitrate and $3 \mathrm{mg} / \mathrm{l}$ nitrite by more than an order of magnitude.

A study on the oral toxicity of plasma-treated edible film coatings conducted in rats found very low toxicity and concluded that the plasma treatment had not generated harmful compounds in the films [58].

\section{Allergen control}

Food allergies affect approximately $10 \%$ of world population; the 'Big 8' food protein sources that trigger allergic reactions are milk, eggs, fish, crustacean/shellfish, treenuts, peanuts, wheat, and soy. The only prevention option available is total avoidance of the food allergen, with individually variable threshold doses. Meinlschmidt and colleagues [59] compared non-thermal technologies for allergen control of soybean and found that maximal efficacy for reduction of soybean protein fraction immuno-reactivity was achieved with direct CP exposure, but that reductions up to $89 \%$ were also achieved using indirect exposure. Segat and colleagues [60] demonstrated that direct CP can unfold whey protein molecules and change its 3-D structures. These recent studies illustrate the potential for $\mathrm{CP}$ as a tool to reduce immunoreactivity of food allergens in foodstuffs and processing environments, and may be particularly suitable for those allergens that prove recalcitrant to standard processing due to their thermostability.

\section{Regulatory aspects}

There is a diversity of global regulatory approaches and processes for applying new technologies to foodstuffs; pertinent details from US and EU jurisdictions only are presented here. In the United States, approval for a new technology such as atmospheric cold plasma potentially has to receive review and primary approval from three federal agencies: Environmental Protection Agency (EPA), Food and Drug Administration (FDA), and United States Department of Agriculture (USDA). Memorandum of Understanding agreements between these agencies allow sharing information and internal communications in the review of new technology reviews for food and food packaging. The US EPA currently has primary regulatory authority for approval of any antimicrobial under the Federal Insecticide, Fungicide, and Rodenticide Act (www.epa.gov/enforcement/federal-insecticide-fungicide- 
and-rodenticide-act-fifra-and-federal-facilities). Before the US EPA approves (registers) a pesticide under FIFRA, the applicant must show efficacy under conditions of use and, among other things, that using the pesticide according to specifications "will not generally cause unreasonable adverse effects on the environment" (www.epa.gov/enforcement/federalinsecticide-fungicide-and-rodenticide-act-fifra-and-federal-facilities). In the context of applying atmospheric cold plasma in agriculture or food processing this would at a minimum require evidence (i.e., scientific data) that the process delivers a consistent treatment for the most extreme process conditions likely to be encountered without creating any unreasonable risk to man or the environment, taking into account the economic, social, and environmental costs and benefits of the use of any pesticide, or a human dietary risk from residues that result from a use of a pesticide in or on any food inconsistent with the standard under section 408 of the Federal Food, Drug, and Cosmetic Act." (www.epa.gov/enforcement/federal-insecticidefungicide-and-rodenticide-act-fifra-and-federal-facilities). A case study outlining regulatory considerations for approval of a cold plasma process for spoilage prevention of wheat is presented in Box 4.

Historically, the FDA evaluated new technologies as having a direct or indirect impact on the food or package and referred to these as a "food additive". The FDA defines a "food additive" as any substance used in producing, processing, treating, packaging, transporting, or storing food, including ionizing radiation. For new technology this occurs under the Premarket Approval for Food Contact Substance (FCS) and is referred to as the Food Contact Notification (FCN) Program. An applicant will submit a FCN request to FDA for a food contact substance (FCS). Atmospheric cold plasma technology used for treatment of food or food packages would be regulated under the US EPA as a pesticide and then US FDA as a FCS (www.fda.gov/Food/IngredientsPackagingLabeling/PackagingFCS/ucm064161.htm.). Additionally, any use of Atmospheric Cold Plasma in meat, poultry or eggs must get approval from the US Department of Agriculture's Food Safety Inspection Service (USDA-FSIS) which has responsibility for approval of any technology, food additive, and its condition of use in meat, poultry and egg products before it can be used in a USDA-FSIS inspected plant. The European Commission's Food Safety approval for new technology is detailed in the MEMO-15-5875 (http://europa.eu/rapid/press-release_MEMO-15-5875_en.htm). Novel food will only be approved for use in the EU if they do not present a risk to public health, are not nutritionally disadvantageous when replacing a similar food and are not misleading to the consumer. A first step requires a "scientific assessment prior to authorisation to ensure their safety". This is performed by the European Food Safety Authority (EFSA) and is called 
PROmoting METHods for Evidence Use in Scientific assessments (PROMETHEUS). The EFSA convenes an expert scientific panel, collects relevant information and then develops an expert report on the benefits and risks of the technology resulting from a "Novel Food" designation. Those novel food applications that receive "Authorisation" can then be sold in the EU. The "Authorisation" sets out the conditions for the novel food use, their designation as

a
food/food ingredient and labeling requirements (http://onlinelibrary.wiley.com/store/10.2903/j.efsa.2017.4737/asset/efs24737.pdf?v=1\&t=j5 qe9gpf \&s=88c071a4bbcb331ed669e20cfe08557a0509374b).

In the last five years (2012 and 2016) a total of 40 novel food ingredient approvals were made, but only four of these involved new technology: UV [technology] treatment of mushrooms, bread, Baker's yeast, and milk. Currently, uncertainty remains in the EC regulatory approval process for cold plasma technologies due to the lack of definition within the evaluation criteria (e.g., "risk to public health", "nutritionally disadvantageous" and "not misleading to the consumer").

\section{Concluding remarks}

321 Overall, recent research demonstrates that it is possible to harness the efficiency of plasma technology for different applications within different stages of food production by optimizing system design for enhancement of microbiological, physiological and chemical quality characteristics of different types of foods. Excitingly, the modification of chemical structures within foods has been demonstrated and provides an avenue for adding value to byproducts waste streams, discovery of functional properties of foods as well as safety in terms of reducing immunoreactivity. There is a need to fully assess the benefits and risks of standalone cold plasma unit processes or their integration as a processing chain, and what the economic, ecological and consumer benefits and acceptability are (see Outstanding Questions). Within primary food production, more research is required to address long-term and multi-generation effects of plasma on seeds and plant growth to produce sustainable foods. The increasing knowledge of the ongoing cold plasma mediated effects within biological systems that has emerged from plasma medicine research, has relevance to plant tissues, insects and cells which are also living systems. This exposes the need to understand the biochemical interactions in detail in each whole system to determine and control the optimum plasma process designs for agriculture and food.

\section{Box 1}


340 Cold plasma (CP) technology finds applications during different stages of agricultural food

341 production. Depending on the product or point of application, plasma treatment may be:

342 - Direct CP, applied to food products in bulk and integrated into the food-processing

343 stream has been proposed for a conveyor belt system [61].

344 - Indirect CP through application of plasma-activated water (PTW) in the form of 345 washes, sprays or mists, can be used for decontamination of fresh produce or processing 346 equipment where liquid disinfectants are currently employed [62-64].

347 - In-package CP discharge generated inside a sealed food package provides 348 decontamination through the action of reactive species over an extended time period and 349 mitigates against recontamination $[65,66]$. This is particularly beneficial for ready-to-eat 350 foods or may be combined with modified atmosphere packaging for meat or fish products.

351 A majority of studies of CP have focused on fresh produce intended for uncooked 352 consumption. The nature of these foods and the demand for fresh and minimally processed 353 foods requires technologies that provide non-thermal treatment with minimal detrimental 354 impact and/or improvement in product quality. Antimicrobial efficacy needs to be balanced 355 with color loss and damage to delicate plant structures especially for ROS-rich discharges 356 [67].

357 CP has been applied for decontamination of dried spices and nuts such as almonds. Cooked

358 food products are less common targets for plasma applications since the cooking procedure 359 itself serves as a decontamination step and the advantage of low temperature treatment 360 offered by cold plasma is not required. The application of plasma to liquid food products 361 such as juice or milk has been studied with regards to decontamination of natural microflora 362 or challenge organisms and products quality preservation [68,69]. Foods with high protein 363 and/or lipid content pose a greater challenge to cold plasma applications both in terms of the 364 efficacy of microbial decontamination and changes to the product quality. A high protein 365 content greatly reduces the antimicrobial efficacy due to scavenging of plasma reactive 366 species as well as allowing microorganisms to recover and regrow more easily post-treatment [70]. Meat products with high lipid content are prone to lipid peroxidation, which can affect product appearance and quality. Application to fish foods also poses challenges in terms of quality retention [71].

\section{Box 2}

372 Selecting cold plasma treatment operating parameters to enhance food safety 
373 The WHO Initiative to Estimate the Global Burden of Foodborne Diseases provided a first 374 estimate on global foodborne disease incidence, mortality, and disease burden in 2015 and 375 reported approximations of 600 million foodborne illnesses and 420,000 deaths globally in 376 2010. Whilst foodborne disease-outbreaks are particularly widespread in the developing 377 world, they still present considerable public health risk in the developed world despite a 378 plethora of food safety measures and hygiene practices. Microorganisms adapt, consumption 379 and trade patterns change and processing errors occur. Microbial pathogens such as 380 Salmonella, Escherichia coli, Listeria and Hepatitis A virus figured strongly in the cases of multi-state disease outbreaks in the US reported by the CDC for 2016 and resulted from products such as fresh or frozen fruit and vegetables, meat or dairy products, while Campylobacterioisis associated with poultry and Salmonellosis presented the predominant outbreaks in Europe. Experimental studies with cold plasma have shown efficient inactivation of pathogenic bacteria such as Salmonella, E. coli, Listeria or surrogate viruses in liquid media, on surfaces or in food models [72]. If cold plasma is considered a food process, it is important to consider the key risks and how processing parameters interact with those risks to arrive at a risk appropriate intervention. To date, key plasma processing variables responsible for efficient inactivation of these microorganisms with 'dry' cold plasma systems have included voltage level, AC waveform frequency, frequency of treatment, treatment time, species retention post treatment and working gas composition. Generally, longer treatment duration, higher frequency and voltage levels can significantly increase antibacterial properties of treatment. The size and the geometry of plasma system is also among essential treatment parameters, influencing the decontamination efficiency of resulting plasma discharge and should be considered in optimization studies with respect to different products for successful integration of this technology in food production lines. For example, a large volume treatment chamber may reduce the total density of plasma reactive species and the probability of collision of these reactive species with inoculated produce, thereby impacting antimicrobial efficacy. As for any surface decontamination technology, the microbiological target, produce surface complexity, geometries and surface area are the main restricting factors limiting CP microbial inactivation process. In addition, disinfection can become less effective against bacterial biofilms associated with rough surfaces of produce and against bacterial cells internalised in produce tissue.

\section{Box 3}


407 Pesticide degradation and agricultural environmental remediation

408 Cold plasma can degrade pesticide residues for a number of different organochlorine and 409 organophosphorus pesticide compounds and on various substrates. Key to the degradation 410 process is the multitude of reactive species in the plasma discharge including $\mathrm{H}_{2} \mathrm{O}_{2}, \mathrm{O}_{3}, \mathrm{O}, \mathrm{H}$, $411 \mathrm{OH}$ radicals, which can be employed for an advanced oxidation process. Pesticide residues in 412 water and on strawberries were significantly reduced by high voltage in-package Dielectric 413 barrier discharge (DBD) discharge after 5-8 min of treatment [73,74]. Analysis of plasmatreated samples suggested the generation of degradation products with simpler chemical groups and lower toxicity than the parent compound. Thus, cold plasma treatment of agrochemicals on food surfaces offers potential for zero-residue clean labels and reduced consumer risk.

With regard to air pollution control, Ye and colleagues [75] successfully utilized continuous direct-current corona discharge plasma for disinfection of air contaminated with Penicillium, suggesting that this technology is a promising technique to control postharvest mold rots during cold storage or to prevent contamination of a controlled growth environment. This offers great potential for safety and sustainability in intensive fresh food production where pesticide alternatives are sought, but where cross contamination is easy with enormous cost implications.

$\mathrm{CP}$ technology has drawn increasing attention for soil remediation contaminated with organic compounds generated from industrial waste emission, agricultural production and atmospheric deposition [76]. The soil remediation from non-aqueous phase organic liquids (NAPLs) (the main contaminants of soil and groundwater environment) is a challenge since they tend to sink in groundwater systems, with complex dispersal and plume patterns. Depending on NAPL composition, atmospheric pressure DBD CP removed high concentrations of NAPL within minutes [77]. Approximately 94\% of glyphosate (nonselective herbicide) was degraded within 45 min of DBD plasma treatment with no phytotoxic effect of the treated soil leachate observed on wheat seed germination and seedling growth [80]. CP DBD has been shown to degrade persistent organic pollutants (POPs) in soil, with removal of polychlorinated biphenyls (PCBs), acid scarlet GR and dichlorodiphenyltrichloroethane (DDT). This presents a viable approach to remediate recalcitrant POPs with high efficiency [78].

\section{Box 4}

\section{Case Study}




\section{Regulatory aspects: Cold Plasma control of wheat spoilage}

442 The process of getting regulatory review and approval in the United States for a new 443 technology is process specific and claim dependent. For example, if one applied for approval 444 of a new dielectric barrier discharge (DBD) direct plasma treatment for whole wheat grain 445 with the claim of spoilage reduction; initial pre-paperwork regulatory meetings are needed to 446 define the specific experimentation, data analysis and post-data analysis protocols required. 447 For cereal grains such as wheat, regulatory oversight resides with USDA-FGIS 448 (https://www.gipsa.usda.gov/fgis/fgis.aspx). However, they do not provide a safety opinion 449 or effectiveness evaluation of a new technology. This is the task of the US EPA (treatment 450 efficacy) and US FDA (product labeling and residue determination). Thus, the initial

451 452 meeting for this new DBD technology use in wheat requires discussion amongst three agencies to determine what data each needs to adequately inform their regulatory decisions and ultimately approval of the process. Based on the intended benefit(s) being claimed and the evaluations completed by US EPA and US FDA, FGIS may allow approval with no declaration or may require labeling of the finished product receiving the plasma treatment (depending on FDA findings regarding residues or whether the process is classified to result in an indirect food additive). Also, if there are measureable deleterious effects on the grain performance, the agencies may decide to place strict limits on its conditions of use and require specific certification or residue testing. US EPA has regulatory authority for efficacy of treatment process and assessment of any environmental impact resulting from the technology. The FDA has the responsibility to determine if the treatment process results in any potential changes to the food and if so, whether these changes are substantive and need to be labeled. Examples of the types of data needed for regulatory evaluation include controlled studies on efficacy for the most difficult product to treat while achieving the targeted efficacy with corresponding quality, chemical, and sensory changes. Additionally, if the claims result in an improved nutrition, enhanced safety (e.g., pasteurization or sterilization), or other marketable benefits, then additional studies and additional regulatory data are required. For example, pasteurization requires identifying the pathogenic organism most resistant to the technology commonly found within or on the product, with demonstrated consistent reductions that meet the regulatory definition of pasteurization. Thus, the emphasis on resistance profiles and mechanistic insights from microbiology studies using cold plasma.

(1)




\section{References}

4761 Devine, CE. (2016) Food Products and Ingredients: Primary Food Production.

477 Reference Module in Food Science. 1 - 15. http://dx.doi.org/10.1016/B978-0-08$478 \quad 100596-5.03450-8$

4792 Schnabel, U. et al. (2012) Decontamination of Microbiologically Contaminated Seeds 480 by Microwave Driven Discharge Processed Gas. J. Agr. Sci. Tech. 1, 99-105.

4813 Dasan, BG. et al. (2016) Decontamination of Aspergillus flavus and Aspergillus parasiticus spores on hazelnuts via atmospheric pressure fluidized bed plasma reactor. Int J Food Microbiol. 216, 50-59.

4844 Butscher, D. et al. (2016) Plasma inactivation of bacterial endospores on wheat grains and polymeric model substrates in a dielectric barrier discharge. Food Control. 60, 636-645.

4875 Butscher, D. et al. (2016) Plasma inactivation of microorganisms on sprout seeds in a dielectric barrier discharge. Int J Food Microbiol. 238, 222-232.

Mitra, A. et al. (2014) Inactivation of Surface-Borne Microorganisms and Increased Germination of Seed Specimen by Cold Atmospheric Plasma. 645-653.

491

Puligundla, P. et al. (2017) Effect of corona discharge plasma jet treatment on decontamination and sprouting of rapeseed (Brassica napus L .) seeds. Food Control. $71,376-382$.

Dasan, BG. et al. (2016) Inactivation of a $\mathrm{fl}$ atoxigenic fungi (Aspergillus spp.) on granular food model, maize, in an atmospheric pressure fluidized bed plasma system. Food Control. 70, 1-8.

4979 Filatova, I. et al. (2013) Plasma seeds treatment as a promising technique for seed germination improvement. ICPIG. 4-7.

49910 Henselová, M. et al. (2012) Growth, anatomy and enzyme activity changes in maize 500 roots induced by treatment of seeds with low-temperature plasma. Biologia (Bratisl). 67/3, 490-497.

50211 Sera, B. et al. (2017) Seed Germination and Early Growth Responses to Seed Pretreatment by Non-thermal Plasma in Hemp Cultivars ( Cannabis sativa L .). Plasma Chem Plasma Process. 37, 207-221.

50512 Stolárik, T. et al. (2015) Effect of Low-Temperature Plasma on the Structure of Seeds, Growth and Metabolism of Endogenous Phytohormones in Pea (Pisum sativum L.). Plasma Chem Plasma Process. 35, 659-676.

50813 Šerá, B. et al. (2012) How various plasma sources may affect seed germination and 
growth. IEEE. 1365-1370.

4 Iranbakhsh, A. et al. (2017) Non-thermal plasma modified growth and physiology in Triticum aestivum via generated signaling molecules and UV radiation. Biol Plant. 17.

5 Ling, L. et al. (2014) Effects of cold plasma treatment on seed germination and seedling growth of soybean. Sci Rep. 4, 1-7.

6 Jiang, J. et al. (2014) Effect of Cold Plasma Treatment on Seed Germination and Growth of Wheat. Plasma Sci Technol. 16, 54-58.

17 Bormashenko, E. et al. (2012) Cold Radiofrequency Plasma Treatment Modifies Wettability and Germination Speed of Plant Seeds. Sci Rep. 2:741, 1-8.

8 Mildaziene, V. et al. (2016) Response of Perennial Woody Plants to Seed Treatment by Electromagnetic Field and Low-Temperature Plasma. Bioelectromagnetics. doi:10.1002/bem.22003

19 Sivachandiran, L. and Khacef A. (2016) Enhanced seed germination and plant growth by atmospheric pressure cold air plasma : combined effect of seed and water treatment. RSC Adv. 7, 1822-1832.

Misra, NN. et al. (2016) Cold plasma interactions with enzymes in foods and model systems. Trends Food Sci Technol. 55, 39-47.

1 Park, DP. et al. (2013) Reactive nitrogen species produced in water by nonequilibrium plasma increase plant growth rate and nutritional yield. Curr Appl Phys. 13, S19-S29.

22 Takaki, K. et al. (2013) Improvements in plant growth rate using underwater discharge. J Phys Conf Ser 7th Int Conf Appl Electrost. 418, 1-7.

3 El-aziz, MFA. et al. (2014) Non thermal plasma for control of the Indian meal moth , Plodia interpunctella ( Lepidoptera : Pyralidae ). J Stored Prod Res. 59, 215-221.

24 Mohammadi, S. et al. (2015) The effect of non-thermal plasma to control of stored product pests and changes in some characters of wheat materials. J Biodivers Environ Sci. 7, 150-156.

Bosch, L. et al. (2017) Plasma-Based Degradation of Mycotoxins Produced by Fusarium, Aspergillus and Alternaria Species. Toxins (Basel). 9, 1-12.

6 Shi, H. et al. (2017) Reduction of Aflatoxin in Corn by High Voltage Atmospheric Cold Plasma Reduction of Aflatoxin in Corn by High Voltage Atmospheric Cold Plasma. Food Bioprocess Technol. DOI 10.100,. doi:10.1007/s11947-017-1873-8

27 Pankaj, SK. et al. (2014) Applications of cold plasma technology in food packaging. 
Trends Food Sci Technol. 35, 5-17.

8 Moon, A. et al. (2016) Feasibility study of atmospheric-pressure plasma treated air gas package for grape â $€^{\mathrm{TM}} \mathrm{s}$ shelf-life improvement. Curr Appl Phys. 16, 440-445.

9 Tappi, S. et al. (2016) Cold plasma treatment for fresh-cut melon stabilization. Innov Food Sci Emerg Technol. 33, 225-233.

Wang, J. et al. (2016) Influence of in-package cold plasma treatment on microbiological shelf life and appearance of fresh chicken breast fillets. Food Microbiol. 60, 142-146.

31 Patange, A. et al. (2017) Controlling Brochothrix thermosphacta as a spoilage risk using in- package atmospheric cold plasma. Food Microbiol. 66, 48-54.

2 Bourke, P. et al. (2017) Microbiological interactions with cold plasma. J Appl Microbiol. doi:10.1111/jam.13429

3 Smet, C. et al. (2017) Impact of food model (micro)structure on the microbial inactivation efficacy of cold atmospheric plasma. Int J Food Microbiol. 240, 47-56.

34 Ziuzina, D. et al. (2015) Cold plasma inactivation of internalised bacteria and biofilms for Salmonella enterica serovar Typhimurium, Listeria monocytogenes and Escherichia coli. Int J Food Microbiol. 210, 53-61.

5 Smet, C. et al. (2016) Influence of food intrinsic factors on the inactivation ef fi cacy of cold atmospheric plasma: Impact of osmotic stress, suboptimal $\mathrm{pH}$ and food structure. Innov Food Sci Emerg Technol. 38, 393-406.

36 Choi, S. et al. (2017) Effect of corona discharge plasma on microbial decontamination of dried squid shreds including physico-chemical and sensory evaluation. LWT - Food Sci Technol. 75, 323-328.

37 Pasquali, F. et al. (2016) Atmospheric cold plasma process for vegetable leaf decontamination : A feasibility study on radicchio ( red chicory, Cichorium intybus L .). Food Control. 60, 552-559.

8 Fröhling, A. et al. (2012) Indirect plasma treatment of fresh pork : Decontamination ef fi ciency and effects on quality attributes. 16, 381-390.

39 Bermúdez-aguirre, D. et al. (2013) Effect of atmospheric pressure cold plasma (APCP) on the inactivation of Escherichia coli in fresh produce. Food Control. 34, 149-157.

40 Ma, R. et al. (2015) Non-thermal Plasma-Activated Water Inactivation of Food-Borne Pathogen on Fresh Produce. J Hazard Mater. doi:10.1016/j.jhazmat.2015.07.061

41 Surowsky, B. et al. (2013) Cold plasma effects on enzyme activity in a model food system. Innov Food Sci Emerg Technol. 19, 146-152. 
57742 Lacombe, A. et al. (2015) Atmospheric cold plasma inactivation of aerobic 578 microorganisms on blueberries and effects on quality attributes. Food Microbiol. 46, $579 \quad 479-484$.

58043 Shi, X. et al. (2011) Effect of Low-Temperature Plasma on Microorganism 581 Inactivation and Quality of Freshly Squeezed Orange Juice Effect of Low-Temperature 582 Plasma on Microorganism Inactivation and Quality of Freshly Squeezed Orange Juice. 583 doi:10.1109/TPS.2011.2142012

58444 Ramazzina, I. et al. (2015) Effect of cold plasma treatment on physico-chemical 585 parameters and antioxidant activity of minimally processed kiwifruit. Postharvest Biol 586 Technol. 107, 55-65.

58745 Bahrami, N. et al. (2016) Cold plasma: A new technology to modify wheat flour 588 functionality. FOOD Chem. 202, 247-253.

58946 Thirumdas, R. et al. (2016) Influence of low pressure cold plasma on cooking and 590

591 textural properties of brown rice. Innov Food Sci Emerg Technol. 37, 53-60.

592 Thirumdas, R. et al. (2017) Functional and rheological properties of cold plasma treated rice starch. Carbohydr Polym. 157, 1723-1731. Park, JH. et al. (2015) Effect of nanosecond-pulsed plasma on the structural 595 modification of biomolecules. RSC Adv. doi:10.1039/C5RA04993H Zhang, H. et al. (2015) Effects and Mechanism of Atmospheric-Pressure Dielectric Barrier Discharge Cold Plasma on Lactate Dehydrogenase ( LDH ) Enzyme. 1-12.

59750 Okazaki, Y. et al. (2014) Direct exposure of non equilibrium atmospheric pressure 598 plasma confers simultaneous oxidative and ultraviolet modifications in biomolecules. $J$ Clinincal Biochem Nutr. 55, 207-215.

60051 Keidar, M. (2015) Plasma for cancer treatment. Plasma Sources Sci Technol. 33001, 60133001.

60252 Kalghatgi, S. et al. (2011) Effects of Non-Thermal Plasma on Mammalian Cells. PLoS $603 \quad$ One. 6, 1-11.

60453 Boxhammer, V. et al. (2013) Investigation of the mutagenic potential of cold 605 atmospheric plasma at bactericidal dosages. Mutat Res / Genet Toxicol Environ $606 \quad$ Mutagen. 753, 23-28.

60754 Boehm, D. et al. (2016) Cytotoxic and mutagenic potential of solutions exposed to 608 cold atmospheric plasma. Nat Publ Gr. 1-14.

60955 Wende, K. et al. (2016) Mutation Research / Genetic Toxicology and Environmental 610 Mutagenesis Risk assessment of a cold argon plasma jet in respect to its mutagenicity. 
611

$612 \quad 56$

613

614

615

616

617

618

619

620

621

622

623

624

625

626

627

628

629

630

631

$632 \quad 63$

633

634

635

636

637

$638 \quad 65$

639

640

$641 \quad 66$

642

643

$644 \quad 67$

Mutat Res - Genet Toxicol Environ Mutagen. 798-799, 48-54.

56 Lukes, P. et al. (2014) Aqueous-phase chemistry and bactericidal effects from an air discharge plasma in contact with water: evidence for the formation of peroxynitrite through a pseudo-second-order post-discharge reaction of $\mathrm{H} 2 \mathrm{O} 2$ and $\mathrm{HNO}$. Plasma Sources Sci Technol. 15019,. doi:10.1088/0963-0252/23/1/015019

57 Jung, S. et al. (2015) The use of atmospheric pressure plasma-treated water as a source of nitrite for emulsion-type sausage. Meat Sci. 108, 132-137.

58 Han, SH. et al. (2016) Oral Toxicity of Cold Plasma-Treated Edible Films for Food Coating. J Food Sci. 0,. doi:10.1111/1750-3841.13551

59 Meinlschmidt, P. et al. (2016) The effects of pulsed ultraviolet light, cold atmospheric pressure plasma, and gamma-irradiation on the immunoreactivity of soy protein isolate. Innov Food Sci Emerg Technol. 38, 374-383.

60 Segat, A. et al. (2016) Effect of atmospheric pressure cold plasma (ACP) on activity and structure of alkaline phosphatase. Food Bioprod Process. 98, 181-188.

61 Toyokawa, Y. et al. (2017) A new roller conveyer system of non-thermal gas plasma as a potential control measure of plant pathogenic bacteria in primary food production. Food Control. 72, 62-72.

62 Ma, R. et al. (2015) Non-thermal Plasma-Activated Water Inactivation of Food-Borne Pathogen on Fresh Produce. J Hazard Mater. doi:10.1016/j.jhazmat.2015.07.061

3 Shen, J. et al. (2016) Bactericidal Effects against S. aureus and Physicochemical Properties of Plasma Activated Water stored at different temperatures. Nat Publ Gr. doi:10.1038/srep28505

64 Schnabel, U. et al. (2016) Antimicrobial efficiency of non-thermal atmospheric pressure plasma processed water ( PPW ) against agricultural relevant bacteria suspensions. Int J Environ Agric Res. 212-224.

5 Klockow, PA. and Keener KM. (2009) LWT - Food Science and Technology Safety and quality assessment of packaged spinach treated with a novel ozone-generation system. LWT - Food Sci Technol. 42, 1047-1053.

6 Ziuzina, D. et al. (2016) Demonstrating the Potential of Industrial Scale In-Package Atmospheric Cold Plasma for Decontamination of Cherry Tomatoes. Plasma Med. 6, $397-412$.

Baier, M. et al. (2014) Non-thermal atmospheric pressure plasma : Screening for 
645

646

$647 \quad 68$

648

649

650

651

$652 \quad 70$

653

$654 \quad 71$

655

656

657

658

659

660

661

662

663

664

665

666

667

$668 \quad 76$

669

670

671

672

$673 \quad 78$

674

675

676

677

678

gentle process conditions and antibacterial ef fi ciency on perishable fresh produce. Innov Food Sci Emerg Technol. 22, 147-157.

8 Kim, H. et al. (2015) Microbial safety and quality attributes of milk following treatment with atmospheric pressure encapsulated dielectric barrier discharge plasma. Food Control. 47, 451-456.

69 Almeida, FDL. et al. (2015) Effects of atmospheric cold plasma and ozone on prebiotic orange juice. Innov Food Sci Emerg Technol. 32, 127-135.

Han, L. et al. (2016) Controlling Microbial Safety Challenges of Meat Using High Voltage Atmospheric Cold Plasma. Front Microbiol Microbiol. 7, 1-12.

71 Albertos, I. et al. (2017) Effects of dielectric barrier discharge (DBD) generated plasma on microbial reduction and quality parameters of fresh mackerel (Scomber scombrus) fillets. Innov Food Sci Emerg Technol. 0-1.

72 Pignata, C. et al. (2017) A review on microbiological decontamination of fresh produce with nonthermal plasma. J Appl Microbiol. 100, 1-18.

73 Sarangapani, C. et al. (2016) Engineering Pesticide degradation in water using atmospheric air cold plasma. J Water Process Eng. 9, 225-232.

74 Misra, NN. et al. (2014) In-package Nonthermal Plasma Degradation of Pesticides on Fresh Produce. J Hazard Mater. 271, 33-40.

75 Ye, S. et al. (2012) Disinfection of airborne spores of Penicillium expansum in cold storage using continuous direct current corona discharge. Biosyst Eng. 113, 112-119.

6 Zhang, H. et al. (2017) Non-thermal plasma technology for organic contaminated soil remediation: A review. Chem Eng J. 313, 157-170.

77 Aggelopoulos, CA. et al. (2016) Parametric analysis of the operation of a non-thermal plasma reactor for the remediation of NAPL-polluted soils. Chem Eng J. 301, 353361.

78 Wang, T. et al. (2016) Glyphosate contaminated soil remediation by atmospheric pressure dielectric barrier discharge plasma and its residual toxicity evaluation. $J$ Hazard Mater. 320, 539-546. 
681 Figure 1 (Key Figure): Atmospheric Cold plasma (CP) applications to agriculture and foods. 682 Applying CP or plasma activated liquids to the agriculture and food continuum has the 683 potential to increase food safety and quality through non-thermal pasteurization or 684 sterilization of food at harvest, post-harvest or processing stages. This can reduce food 685 waste; efficiently degrade pesticides and mycotoxins as well as inactivate pests. Cold plasma 686 processes may be used to generate nitrate rich substrates, thereby increasing seeds germination and yield.

688

689 Figure 2: Atmospheric Cold plasma (CP) interactions with microbial contaminants and 690 foods.

691 Numerous studies demonstrated that CP has inactivation effects on bacteria via bacterial cell

692 wall/membrane or extracellular polymeric substance disruption or via action on intracellular 693 components, metabolic activity or virulence factors such as prevention of biofilm formation 694 leading to cell death. Besides improving microbiological quality, CP treatment results in 695 enhanced physicochemical, physiological and functional properties of foods.

696

697

\section{Glossary}

699 Biofilms: Biofilms are 3D communities of sessile microorganisms either in mono or mixed 700 populations, which provide protection to microbial contaminants against antimicrobial or decontamination treatments.

702 Cold plasma (CP): is commonly referred to as the fourth state of matter where increases in a material energy levels converts its state from solid to liquid to gas and ultimately to an ionized state of the gas, "plasma", which exhibits unique properties. Cold plasma (CP) is comprised of several excited atomic, molecular, ionic, and radical species, co-existing with numerous reactive species, including electrons, positive and negative ions, free radicals, gas atoms, molecules in the ground or excited state and quanta of electromagnetic radiation (UV photons and visible light). Depending on the generation conditions, plasma can be classified into low-, atmospheric- or high-pressure and also subdivided into thermal and non-thermal (i.e. cold) plasmas. Thermal plasma can be generated by heating the gas to high temperatures, which may exceed several thousands of Kelvins, where all the constituent chemical species electrons and ions exist in a thermodynamic equilibrium. In contrast, non-thermal or cold 
713 plasmas are characterized by non-equilibrium, where cooling of the ions and uncharged 714 molecules is significantly more effective than that of energy transfer from electrons resulting 715 in the gas remaining at a low temperature.

716 Dielectric barrier discharge (DBD): is the electrical discharge between two electrodes 717 separated by an insulating dielectric material

718 Fungal spores: small resistant structures $(2-20 \mu \mathrm{m})$ that serve for fungal reproduction and 719 survival

720 Mycotoxins: secondary metabolites produced by fungi that can cause adverse health effects in both humans and animals. Mycotoxins are mainly produced by 6 genera of fungi: Aspergillus, Penicillium, Fusarium, Alternaria, Claviceps and Stachybotrys occurring in food and feed commodities both pre- and post-harvest. The adverse effects include carcinogenic, mutagenic, estrogenic, nephrotoxic, neurotoxic, hepatotoxic, immunosuppressive and gastrointestinal toxicity.

726 Pesticides: constitute any substance or mixture of substances intended for preventing, destroying, repelling, or mitigating any pests. The term pesticide covers a wide range of compounds including insecticides, fungicides, herbicides, rodenticides, molluscicides, nematicides, plant growth regulators and others. They can also serve as plant regulators, defoliants, or dessicants.

Plasma treated water (PTW): PTW can be obtained by subjecting water to a plasma process. This results in the generation of a wide range of chemically reactive species that can be retained within the water. Plasma treatment can be applied to liquid effluent from various industrial sectors for decontamination purposes, which can refer to plasma processed liquids. Term "plasma activated water" is widely used for plasma medicine applications. Stress hardening: microorganisms adapted to stress, which develop homologous or crossresistance. Continuous exposure of bacteria to sub-lethal stresses may lead to a homologous resistance, i.e. lead to the increased resistance to subsequent applications of the same stress. Foods are complex environments in which microorganisms may face a range of stresses from the food itself (intrinsic stress) or process (extrinsic stress) environment. Cells adapted to stress, hardened, are able to withstand further stresses coming from processing environment or food treatments. For example, E. coli is more resistant to ozone treatment when cells are pre-exposed to acidic environment; L. monocytogenes develops cross resistance to antibiotic treatment due to either an increase in salt concentration, acidic environment or reduced temperature conditions. 


\section{Future reading}

Crandall, SG. and Gilbert, GS. (2017) Meteorological factors associated with abundance of airborne fungal spores over natural vegetation. Atmospheric Environment, 162, 87-99. Demaegdt, H. et al. (2016) Endocrine activity of mycotoxins and mycotoxin mixtures. Food and Chemical Toxicology, 96, 107-116. Bernardes, MFF, et al. (2015) Impact of Pesticides on Environmental and Human. In: A perspective on the potential risks of emerging contaminants to human and environmental health. Environmental Science and Pollution Research, 195 - 233. DOI: 10.1007/s11356015-4896-6.

Alvarez-Ordóñez, A. et al. (2015) The adaptive response of bacterial food-borne pathogens in the environment, host and food: Implications for food safety. International Journal of Food Microbiology, 213, 99-109. 


\section{Trends}

2 - In package decontamination of foods using cold plasma has advanced this technology 3 as a unit process for fresh foods decontamination and shelf -life extension.

4 - Chemical residues of agricultural pesticides of varying structure can be degraded to 5 safe or less toxic structures using cold plasma.

6 - Cold plasma mediated control of contaminants with the promotion of seeds germination and plant growth offers alternatives to current pesticides and fertilizers for agriculture.

- Controlling plasma reactive species formulations in dry and liquid delivery formats advances the potential for understanding and successful translation to multiple points along the agriculture and food sectors.

- Employing predictive microbiology, process optimization tools and a systems approach with controlled reactive species formulations may achieve risk or problem tailored solutions for whole food systems. 


\section{Outstanding questions}

2

3

4

- Can plasma treatment achieve commercial sterility with regards to biological, chemical and allergenic contaminants and result in 'zero residue' products? In the context of 'clean labels' this would provide market advantage and a potential route to consumer acceptance.

- Importantly, in terms of antimicrobial resistance or pesticide resistance - key issues in agriculture and food production - can cold plasma treatment exert long-term selective pressure for resistant microorganisms and pests?

- Can cold plasma treatment lead to increased horizontal gene transfer in the existing microflora?

1 - In terms of multi-species contaminants and microbial ecologies, will cold plasma 2 treatment enhance or decrease the safety or shelf-life profile?

3 - Are cold plasma reactive species and their effects controllable in complex food or 4 environmental matrices?

5 - What are the cold plasma reactive species formulations required to control specific 16 biological or chemical risks?

7 - Can cold plasma deposition lead to chemical cross-contamination of food products?

8 - How does plasma treatment effect the biodegradability of agricultural and food 9 waste?

20 - What are the organoleptic impacts on food formulations? Does it impact taste $1 \quad$ positively or at all?

22 What is the consumer acceptability of this technology for food processing? 


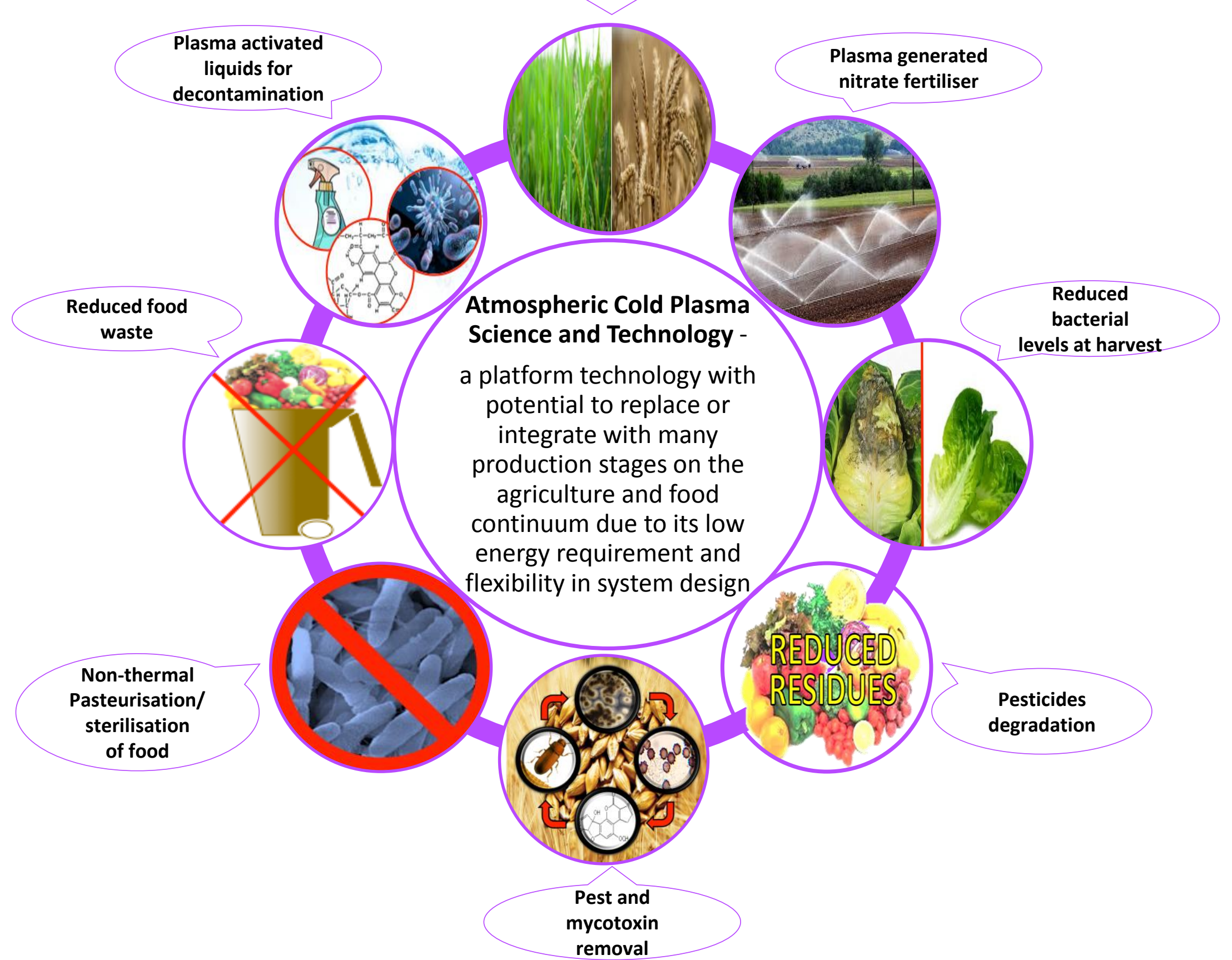




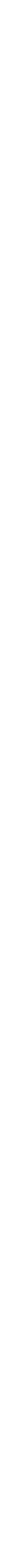

